\title{
The superior fovea triangle approach: a novel safe entry zone to the brainstem
}

\author{
${ }^{*}$ Kaan Yagmurlu, MD, M. Yashar S. Kalani, MD, PhD, Mark C. Preul, MD, and Robert F. Spetzler, MD \\ Department of Neurosurgery, Barrow Neurological Institute, St. Joseph's Hospital and Medical Center, Phoenix, Arizona
}

The authors describe a safe entry zone, the superior fovea triangle, on the floor of the fourth ventricle for resection of deep dorsal pontine lesions at the level of the facial colliculus. Clinical data from a patient undergoing a suboccipital telovelar transsuperior fovea triangle approach to a deep pontine cavernous malformation were reviewed and supplemented with 6 formalin-fixed adult human brainstem and 2 silicone-injected adult human cadaveric heads using the fiber dissection technique to illustrate the utility of this novel safe entry zone. The superior fovea has a triangular shape that is an important landmark for the motor nucleus of the trigeminal, abducens, and facial nerves. The inferior half of the superior fovea triangle may be incised to remove deep dorsal pontine lesions through the floor of the fourth ventricle. The superior fovea triangle may be used as a safe entry zone for dorsally located lesions at the level of the facial colliculus.

https://thejns.org/doi/abs/10.3171/2016.8.JNS16947

KEY WORDS anatomy; brainstem surgery; floor of the fourth ventricle; safe entry zone; superior fovea; surgical technique

$\mathrm{T}$ HE human brainstem, roughly the size of the thumb, carries ascending and descending fiber tracts that are essential for motor and sensory control. The dense packing of these critical structures means that any manipulation of the brainstem is fraught with the risk of causing deficits, albeit temporary in many cases. The eloquence of the brainstem has resulted in most neurosurgeons shying away from approaching lesions that do not abut a pial or an ependymal surface. For deep-seated lesions, several safe entry zones - areas where the brainstem may be traversed with minimal or acceptable morbidityhave been described. ${ }^{7,9}$ Increasingly detailed anatomical knowledge gained from fiber dissections and neuroimaging techniques, such as MRI tractography, can now be used to supplement anatomical intuition to describe the relationship between the lesion and the eloquent white matter tracts and guide the approach ${ }^{12,13}$ to decrease the morbidity associated with resecting intrinsic brainstem lesions. For lesions deep to the floor of the fourth ventricle, 2 safe entry zones have been described: the suprafacial and infrafacial safe entry zones. ${ }^{13}$ However, no safe entry zone has been described to safely reach deep lesions at the level of the facial colliculus. In this report, we describe the superior fovea triangle approach, a novel safe entry zone on the floor of the fourth ventricle, for deep dorsal pontine lesions located at the level of the facial colliculus. We illustrate the utility of this approach with the clinical case of a patient treated for a cavernous malformation, and we supplement this example with anatomical dissections to better define this new safe entry zone.

\section{Methods}

Six formalin-fixed adult human brainstems and 2 adult human cadaveric heads injected with colored silicone were examined. The human brainstems were dissected using fiber dissection techniques with microdissectors under magnifications of $\times 6$ to $\times 40$ with a Zeiss Surgical Microscope (Carl Zeiss AG). The midline suboccipital telovelar approach was used on the heads to approach the superior fovea triangle.

The clinical and radiographic histories of a 68-year-old male patient were reviewed retrospectively to document neurological examination and radiographic parameters.

ABBREVIATIONS $\mathrm{CN}=$ cranial nerve.

SUBMITTED April 13, 2016. ACCEPTED August 25, 2016.

INCLUDE WHEN CITING Published online December 23, 2016; DOI: 10.3171/2016.8.JNS16947.

* Drs. Yagmurlu and Kalani contributed equally to this work. 


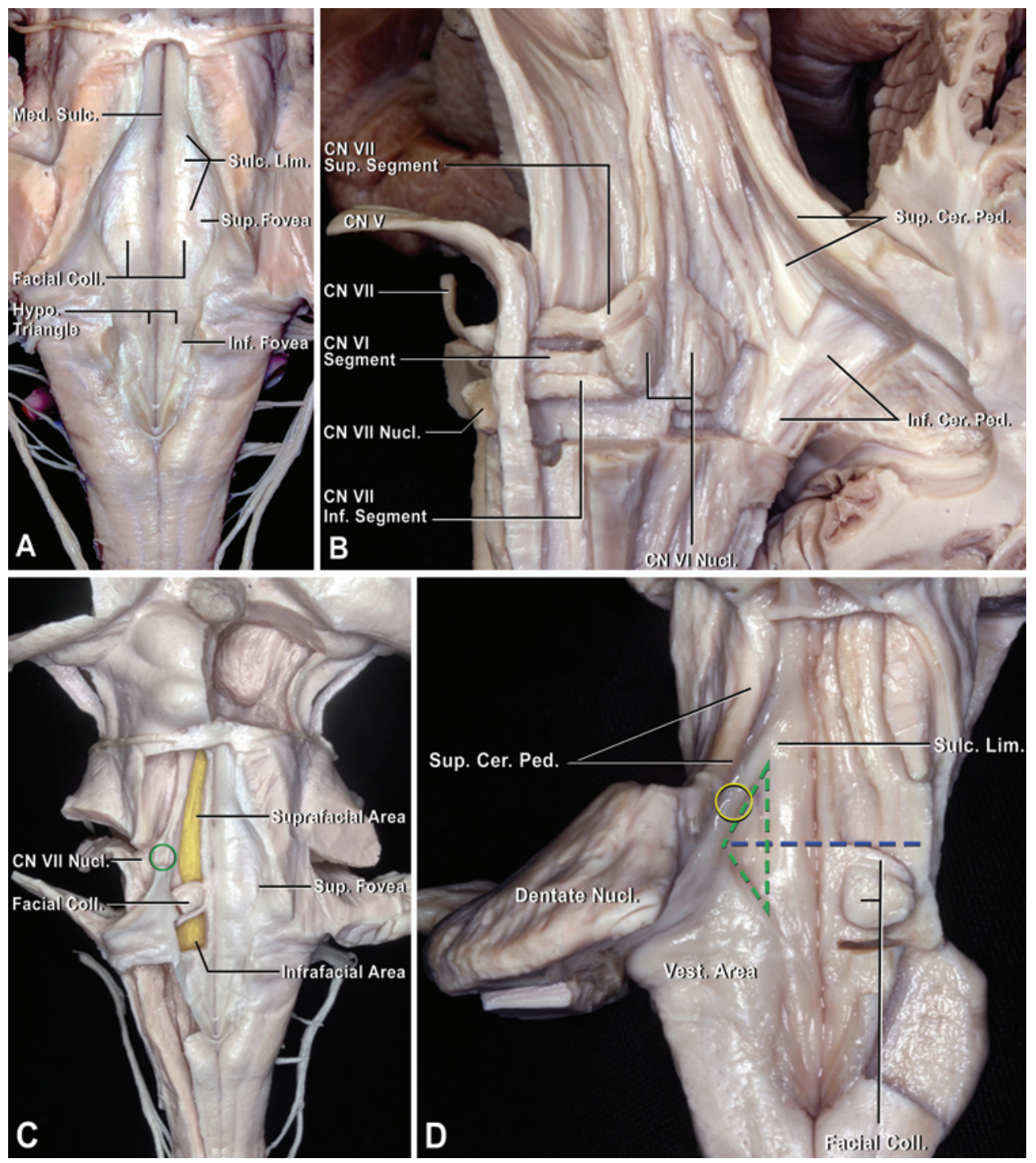

FIG. 1. Superior fovea triangle. A: The sulcus limitans, a lateral sulcus, deepens at 2 points to form dimples called fovea. The superior fovea is located lateral to the facial colliculus, and the inferior fovea is located lateral to the hypoglossal triangle. B: The facial colliculus is formed by the abducens nucleus and the intrapontine segment of the facial nerve. The superior cerebellar peduncle forms the superior half of the lateral wall of the fourth ventricle, and the inferior cerebellar peduncle forms the inferior half of the lateral wall of the fourth ventricle. C: The suprafacial approach, above the facial colliculus, and the infrafacial approach, below the facial colliculus, are used for dorsal pontine lesions. The trigeminal motor and main sensory nuclei (green circle) are located above the upper level of the facial colliculus and the most lateral point of the fourth ventricle. D: The sulcus limitans deepens lateral to the facial colliculus, which is called the superior fovea. The superior fovea has a triangular shape (green dashed triangle): its superolateral edge is formed by the superior cerebellar peduncle, its inferolateral edge is formed by the vestibular area, and its medial edge is formed by the sulcus limitans. The trigeminal motor nuclei (yellow circle) are located deep to the superolateral edge of the superior fovea triangle, and the apex of the triangle is located at the same transverse level as the upper edge of the facial colliculus (blue dashed line). Cer. Ped. = cerebellar peduncle; Coll. = colliculus; Hypo = hypoglossal; Inf. = inferior; Med. Sulc. = median sulcus; Nucl. = nucleus; Sulc. Lim. = sulcus limitans; Sup. = superior; Vest. = vestibular. Dissections prepared by Kaan Yagmurlu, MD. Reproduced with permission from the Rhoton Collection (http://rhoton.ineurodb.org), CC BY-NC-SA 4.0 (http://creativecommons.org/licenses/by-nc-sa/4.0).

\section{Results}

\section{Surgical Anatomy of the Superior Fovea Triangle}

The sulcus limitans deepens at 2 points to form dimples called fovea. The inferior fovea is located lateral to the hypoglossal triangle, and the superior fovea is located lateral to the facial colliculus, which is formed by the abducens nucleus and the intrapontine segment of the facial nerve (cranial nerve [CN] VII) (Figs. 1 and 2). The superior fovea, which has a triangular shape, is located lateral to the facial colliculus. ${ }^{13}$ The superolateral edge of the superior fovea triangle is formed by the superior cerebellar peduncle, the inferolateral edge is formed by the vestibular area, and the medial edge is formed by the sulcus limitans. The apex of the triangle is located at the same transverse level as the upper edge of the facial colliculus. The trigeminal 


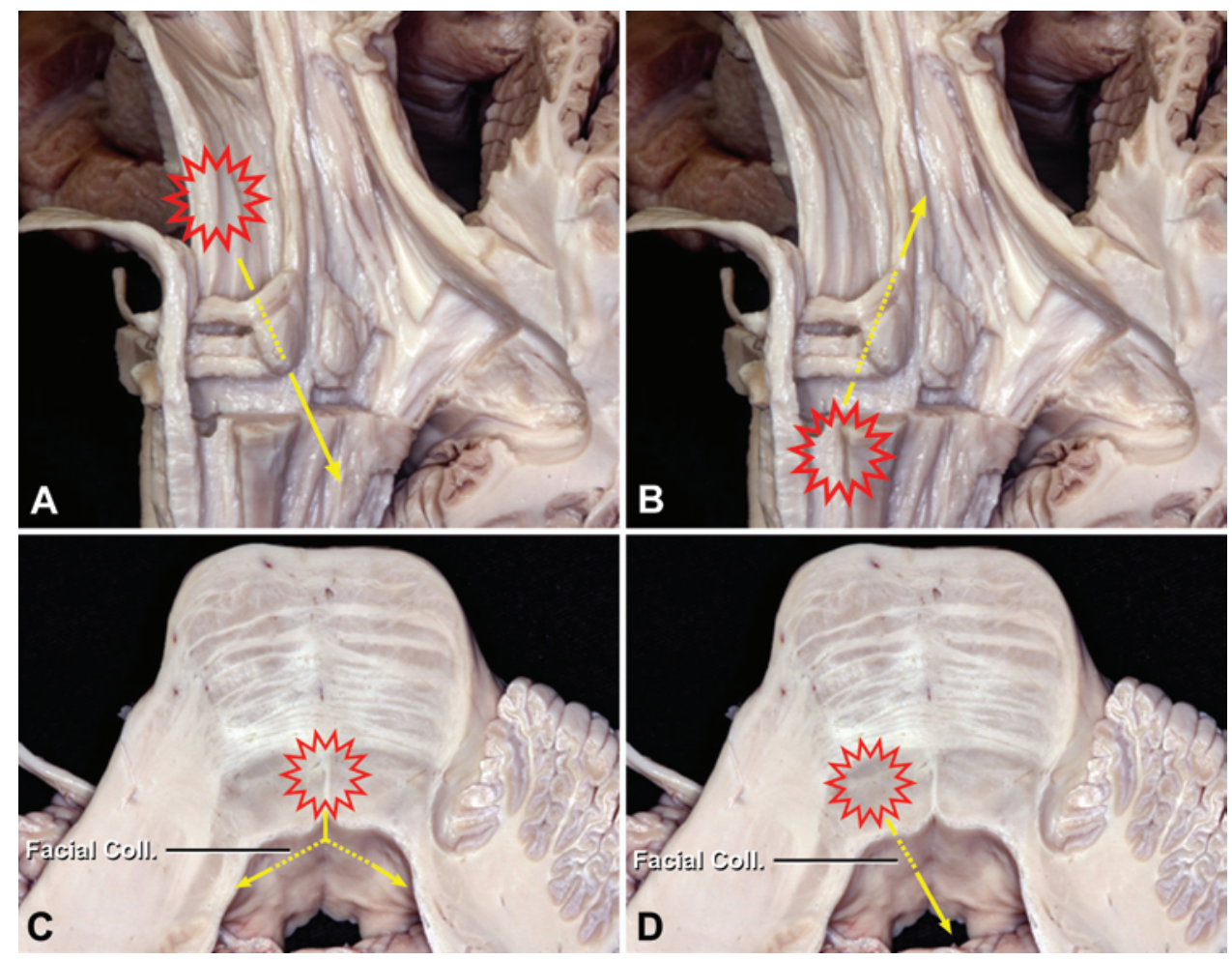

FIG. 2. Possibilities of displacement (arrow) of the facial colliculus according to different lesion locations (starburst). A: A lesion located rostral to the axial level of the facial colliculus pushes the facial colliculus caudally. B: A lesion located caudal to the axial level of the facial colliculus displaces the facial colliculus rostrally. C: A lesion located at the same axial level as the facial colliculus and close to the midline displaces the facial colliculus laterally toward the superior fovea. D: A lesion located at the same axial level as the facial colliculus and laterally displaces the facial colliculus medially. Coll. = colliculus. Dissections prepared by Kaan Yagmurlu, MD. Reproduced with permission from the Rhoton Collection (http://rhoton.ineurodb.org), CC BY-NC-SA 4.0 (http:// creativecommons.org/licenses/by-nc-sa/4.0).

motor and main sensory nuclei sit deep to the superolateral edge of the triangle. In the midline suboccipital telovelar approach (Fig. 3), a vertical incision is made, extending from the level of the apex of the superior fovea triangle to the vestibular area, which is the inferolateral edge of the superior fovea triangle.

\section{Case Report}

A 68-year-old right-handed man presented to our clinical service for evaluation. He had previously experienced sudden-onset left facial weakness and had persistent mild facial weakness (House-Brackmann Grade II). MRI revealed a small, deep, dorsal pontine lesion at the level of the left facial colliculus (Fig. 4A-C).

The patient underwent resection of the lesion using a midline suboccipital telovelar approach. Because of the location of the lesion and the existing mild deficit, and the need to avoid the midline fourth ventricle, a lateral transsuperior fovea opening was made in the brainstem and expanded in a craniocaudal fashion. Although we monitor somatosensory evoked potentials, motor evoked potentials, and specific cranial nerves, we did not perform direct stimulation of the facial colliculus. The lesion was resected in a gross-total fashion (Fig. 4D and E). Postoperatively, the patient experienced worsening of his facial weakness (House-Brackmann Grade IV) but improved over the following 2 months (House-Brackmann Grade II). He was otherwise neurologically unchanged.

\section{Discussion}

Various safe entry zones to the floor of the fourth ventricle have been proposed for removal of intrinsic dorsal pontine lesions. When the lesion reaches the surface of the floor of the fourth ventricle, it creates a natural corridor to the lesion. In the case of an intraaxial lesion that does not reach the surface, the safe entry zones are used to avoid damage to important structures. On the floor of the fourth ventricle, the proposed safe entry zones above the facial colliculus are the suprafacial median sulcus approaches; below the facial colliculus, the infrafacial approach is used for dorsal pontine lesions. ${ }^{2-4,6,8,10,13}$ In addition to these safe entry zones, the superior fovea triangle approach can be used for deep lesions located at the level of the facial colliculus.

Bertalanffy et al. ${ }^{1}$ electrophysiologically stimulated the facial nerve on the surface of the floor of the fourth ventricle to identify the precise location of the distorted facial colliculus intraoperatively in a patient with brainstem lesions. They noted that facial nerve responses have a remarkable variability, even in patients with considerable distance between the facial colliculus and the lesion. For 40 response areas that were mapped, most of the facial nerve 

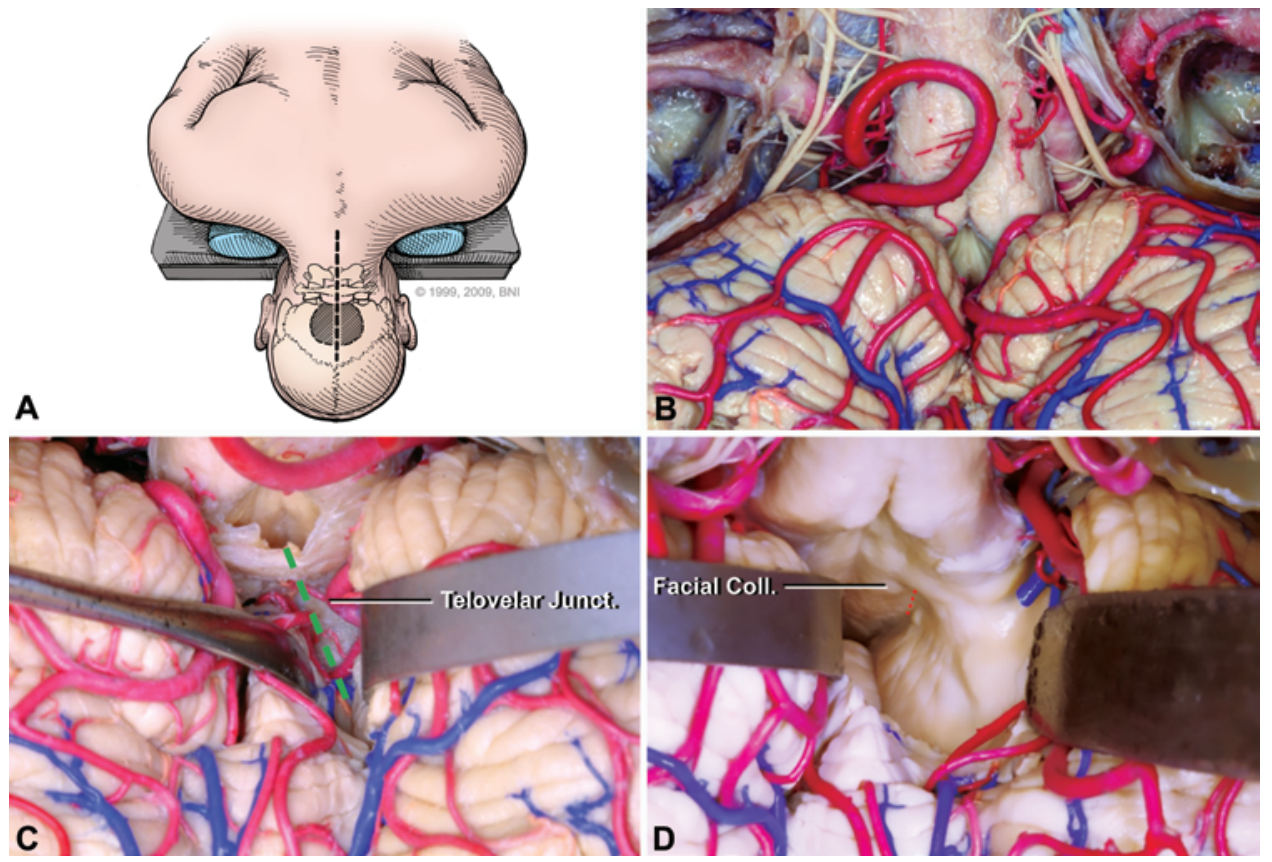

FIG. 3. Midline suboccipital telovelar approach. A and B: The suboccipital midline craniotomy and dural opening $(A)$ expose the dorsal medulla and the cerebellar tonsils (B). C: The telovelar junction is exposed by opening the sulcus between the uvula and the cerebellar tonsil. The telovelar junction is incised (green dashed line) to expose the floor of the fourth ventricle. D: The facial colliculus is a prominence on the fourth ventricle, and the inferior half of the superior fovea triangle is incised (red dotted line). Coll. = colliculus; Junct. = junction. Panel A: Copyright Barrow Neurological Institute, Phoenix, Arizona. Published with permission. Panels B-D: Dissections prepared by Kaan Yagmurlu, MD. Reproduced with permission from the Rhoton Collection (http://rhoton. ineurodb.org), CC BY-NC-SA 4.0 (http://creativecommons.org/licenses/by-nc-sa/4.0).
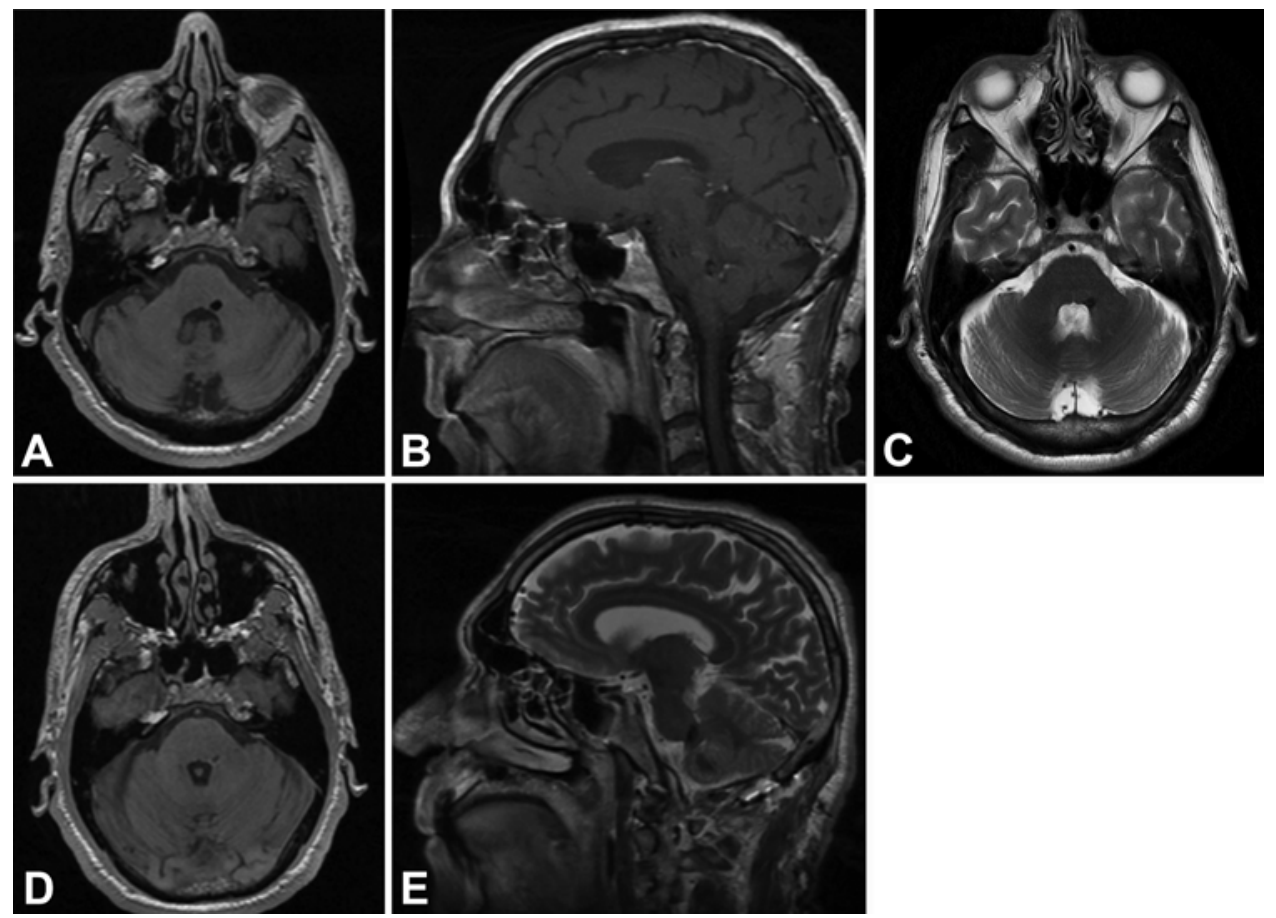

FIG. 4. A 68-year-old male patient presented with acute-onset facial paralysis. A-C: Preoperative axial T1-weighted (A), sagittal T1-weighted (B), and axial T2-weighted (C) MRI studies demonstrating a dorsal pontine cavernous malformation at the level of the facial colliculus. The lesion was approached using a midline suboccipital telovelar approach. The opening in the brainstem was placed laterally at the superior fovea. D and E: Postoperative axial T1-weighted (D) and sagittal T2-weighted (E) MRI studies demonstrating complete resection of the lesion. 
responses were displaced medially and rostrally or caudally, and were less likely to be displaced laterally toward the superior fovea. To avoid complications, Bertalanffy et al. pointed out the importance of using electrical mapping of motor cranial nerves on the floor of the fourth ventricle as well as of using intraoperative monitoring of somatosensory evoked potentials and motor evoked potentials for assessment of the safe entry zones to the brainstem.

The 2-point method is used to determine the safest and shortest route from the pial-ependymal surface of the brainstem to deep lesions. ${ }^{5}$ Whenever possible, the safe entry zones should be combined with the 2-point method to identify the ideal surgical route for deep-seated lesions. In the case presented in this report, the ideal approach was a midline suboccipital telovelar approach ${ }^{11}$ to the floor of the fourth ventricle.

\section{Conclusions}

The superior fovea triangle is an important landmark for the motor nucleus of the trigeminal (CN V), abducens (CN VI), and facial nerves, and it may be used as a safe entry zone for dorsal lesions located at the level of the facial colliculus. In addition, the use of intraoperative stimulation of motor cranial nerves on the floor of the fourth ventricle is helpful in providing safer and more accurate brainstem surgery.

\section{Acknowledgments}

We acknowledge the Neuroscience Publications office at Barrow Neurological Institute for assistance with preparation of this manuscript. All dissections and photography were performed by the first author at the Dr. Albert L. Rhoton Neuro-Microanatomy Lab, Lillian S. Wells Department of Neurosurgery at the University of Florida, and at the Neuroanatomy Laboratory, Barrow Neurological Institute, Phoenix, Arizona.

\section{References}

1. Bertalanffy H, Tissira N, Krayenbühl N, Bozinov O, Sarnthein J: Inter- and intrapatient variability of facial nerve response areas in the floor of the fourth ventricle. Neurosurgery 68 (1 Suppl Operative):23-31, 2011

2. Bogucki J, Czernicki Z, Gielecki J: Cytoarchitectonic basis for safe entry into the brainstem. Acta Neurochir (Wien) 142:383-387, 2000

3. Bricolo A: Surgical management of intrinsic brain stem gliomas. Oper Tech Neurosurg 3:137-154, 2000

4. Bricolo A, Turazzi S: Surgery for gliomas and other mass lesions of the brainstem. Adv Tech Stand Neurosurg 22:261-341, 1995

5. Brown AP, Thompson BG, Spetzler RF: The two-point method: evaluating brain stem lesions. BNI Q 12:20-24, 1996

6. Cantore G, Missori P, Santoro A: Cavernous angiomas of the brain stem. Intra-axial anatomical pitfalls and surgical strategies. Surg Neurol 52:84-94, 1999

7. Cavalcanti DD, Preul MC, Kalani MY, Spetzler RF: Microsurgical anatomy of safe entry zones to the brainstem. J Neurosurg 124:1359-1376, 2015

8. Giliberto G, Lanzino DJ, Diehn FE, Factor D, Flemming KD, Lanzino G: Brainstem cavernous malformations: anatomical, clinical, and surgical considerations. Neurosurg Focus 29(3):E9, 2010

9. Kalani MY, Yagmurlu K, Martirosyan NL, Spetzler RF: The retrosigmoid petrosal fissure transpeduncular approach to central pontine lesions. World Neurosurg 87:235-241, 2016

10. Kyoshima K, Kobayashi S, Gibo H, Kuroyanagi T: A study of safe entry zones via the floor of the fourth ventricle for brain-stem lesions. Report of three cases. J Neurosurg 78:987-993, 1993

11. Rhoton AL Jr: Cerebellum and fourth ventricle. Neurosurgery 47 (3 Suppl):S7-S27, 2000

12. Ulrich NH, Kockro RA, Bellut D, Amaxopoulou C, Bozinov $\mathrm{O}$, Burkhardt JK, et al: Brainstem cavernoma surgery with the support of pre- and postoperative diffusion tensor imaging: initial experiences and clinical course of 23 patients. Neurosurg Rev 37:481-492, 2014

13. Yagmurlu K, Rhoton AL Jr, Tanriover N, Bennett JA: Threedimensional microsurgical anatomy and the safe entry zones of the brainstem. Neurosurgery 10 (Suppl 4):602-620, 2014

\section{Disclosures}

The authors report no conflict of interest concerning the materials or methods used in this study or the findings specified in this paper.

\section{Author Contributions}

Conception and design: all authors. Acquisition of data: Yagmurlu, Kalani. Analysis and interpretation of data: all authors. Drafting the article: Yagmurlu, Kalani. Critically revising the article: Spetzler, Preul. Reviewed submitted version of manuscript: all authors. Statistical analysis: Yagmurlu, Kalani. Administrative/ technical/material support: Spetzler, Kalani, Preul. Study supervision: Spetzler.

\section{Correspondence}

Robert F. Spetzler, c/o Neuroscience Publications, Barrow Neurological Institute, St. Joseph's Hospital and Medical Center, $350 \mathrm{~W}$ Thomas Rd., Phoenix, AZ 85013. email: neuropub@ dignityhealth.org. 